\title{
Case Report \\ Pertussis Reinfection in an Adult: A Cause of Persistent Cough Not to Be Ignored
}

\author{
Theocharis Koufakis, ${ }^{1}$ Anastasia Paschala, ${ }^{2}$ and Dimitrios Siapardanis ${ }^{3}$ \\ ${ }^{1}$ Department of Internal Medicine, General Hospital of Larissa, 1 Tsakalof Street, 41221 Larissa, Greece \\ ${ }^{2}$ George Papanikolaou General Hospital, Exochi, 57010 Thessaloniki, Greece \\ ${ }^{3}$ Department of Internal Medicine, St. Josef Hospital, Robert-Koch-Straße 16, 42781 Haan, Germany \\ Correspondence should be addressed to Theocharis Koufakis; thkoyfak@hotmail.com
}

Received 12 April 2017; Revised 8 June 2017; Accepted 13 June 2017; Published 13 July 2017

Academic Editor: Julian Thomas

Copyright (c) 2017 Theocharis Koufakis et al. This is an open access article distributed under the Creative Commons Attribution License, which permits unrestricted use, distribution, and reproduction in any medium, provided the original work is properly cited.

\begin{abstract}
Pertussis is traditionally considered as a disease of the childhood; however, accumulating evidence suggests a stable increase of its incidence among adults and adolescents, during the last decades. Despite the fact that reinfection after natural disease or vaccination is not uncommon, the index of clinical suspicion of pertussis diagnosis in adults remains low. In this article, we report a case of pertussis reinfection 30 years after natural infection, which was complicated by pneumonia, and we discuss our diagnostic and therapeutic approach, aiming to raise clinicians' degree of suspicion regarding pertussis diagnosis in adults. Prompt recognition and appropriate therapy of adult patients can result in the effective control of the symptoms, prevention of severe complications, and spread of the infection to children; thus, they are of great clinical and public health importance.
\end{abstract}

\section{Introduction}

Pertussis (also known as whooping cough) is a respiratory tract infection, caused mainly by the gram-negative, aerobic, pathogenic, encapsulated coccobacillus Bordetella pertussis. Still, a similar to pertussis clinical entity caused by Bordetella parapertussis has been also described in humans [1]. The disease is considered to be highly contagious. Its main clinical manifestation is the paroxysmal cough, which, in some cases, can be extremely torturous for the patient, since it can last for weeks and be accompanied by apnea, exhaustion, and posttussive vomiting [2].

Despite the fact that most countries have adopted extended vaccination programs against the disease, pertussis continues to be a significant public health challenge. Important differences in mortality rates among very young, unimmunized infants, depending on whether or not adequate supportive care is available, have been observed [3]. Although it is traditionally considered as a disease of the childhood, accumulating evidence suggests a stable increase of pertussis incidence among adults and adolescents, during the last decades [4].
Reinfection after natural disease or vaccination is not uncommon [5]; however, the index of clinical suspicion of pertussis diagnosis in adults remains low. While the duration of protection in immunized subjects seems to be shorter for those immunized with acellular pertussis vaccines, protection offered by whole-cell pertussis vaccines is not lifelong [5]. Immunological protection against the typical disease starts to wane 3 to 5 years and nearly disappears approximately 12 years after vaccination [6]. In this article, we report a case of pertussis reinfection 30 years after natural infection, which was complicated by pneumonia, and we discuss our diagnostic and therapeutic approach, aiming to raise clinicians' degree of suspicion regarding pertussis diagnosis in adults.

\section{Case Presentation}

A 40-year-old female patient presented with complaints of prolonged, paroxysmal cough for the last 4 weeks. The cough was followed by gasping for breath and rarely vomiting. She was obese (body mass index $41 \mathrm{~kg} / \mathrm{m}^{2}$ ) and had a history of recently diagnosed Diabetes Mellitus Type 2, for which 
TABLE 1: Patient's main laboratory findings on presentation. Notably, leukocytosis is generally uncommon in adults and even in the absence of it, the diagnosis of pertussis should not be excluded.

\begin{tabular}{lc}
\hline Parameter (units, reference range) & Value \\
\hline White blood cell $\left(10^{3} / \mu \mathrm{L}, 4-11\right)$ & 10.2 \\
CRP $(\mathrm{mg} / \mathrm{dl},<0.8)$ & 3.2 \\
Alanine aminotransferase $(\mathrm{IU} / \mathrm{L},<40)$ & 24 \\
Lactate dehydrogenase $(\mathrm{IU} / \mathrm{L},<350)$ & 141 \\
Creatine phosphokinase $(\mathrm{IU} / \mathrm{L},<100)$ & 74 \\
\hline
\end{tabular}

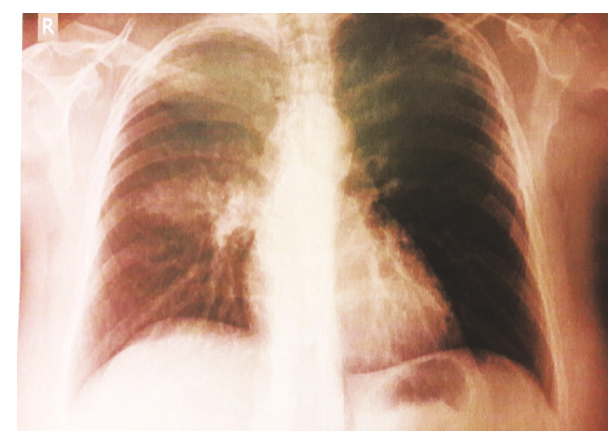

Figure 1: Patient's chest X-ray demonstrating a right upper lobe consolidation.

she was on metformin, $500 \mathrm{mg}$, orally, twice a day. She had received a 7-day course of amoxicillin-clavulanic acid (1000 mg, twice a day) and inhaled fluticasone, with no clinical response.

On physical examination, she was looking fatigued and had low grade fever $\left(37.4^{\circ} \mathrm{C}\right)$ and mild sinus tachycardia (95 beats per minute), while her blood pressure and oxygen saturation were normal $(140 / 90 \mathrm{mmHg}$ and $98 \%$, resp.). On lung auscultation, chest sounded clear. Her initial laboratory evaluation was normal, apart from elevated C-Reactive Protein (CRP) (Table 1). However, her chest X-ray demonstrated a right upper lobe consolidation (Figure 1).

After looking back at her personal medical record, we discovered that the patient had undergone a pertussis infection, at the age of ten. The original diagnosis had been confirmed by a positive result for Bordetella pertussis nasopharyngeal aspirate specimen culture and the disease had been treated with erythromycin. She had been never vaccinated against the disease, as confirmed by her medical record documentation and the patient's mother who was also interviewed.

Further laboratory work-up revealed negative urine antigen tests for Streptococcus pneumoniae and Legionella pneumophila, negative immune serologic tests for Mycoplasma pneumoniae, Chlamydophila pneumoniae, and Coxiella burnetii, and negative urine and blood cultures. Nasopharyngeal samples for culture and PCR for pertussis were not taken early in the course of infection, due to lack of suspicion of pertussis. The diagnosis of acute pertussis infection was established by high titer (120 ELISA units/ml) of IgG antibodies against Pertussis Toxin (PT). The serological testing was performed in a private laboratory, with a commercial assay for measuring
IgG anti-PT antibodies (Savyon Diagnostics, Ashdod, Israel), accredited according to European (EN) 15189 standard, as proposed by the European Center for Disease Prevention and Control (ECDC) guidelines for pertussis laboratory diagnosis [7]. The patient was treated with a 7-day clarithromycin course $(500 \mathrm{mg}$, twice a day), which resulted in the control of her symptoms within the next 2 weeks. No other similar case was reported among the patients' relatives or coworkers; therefore, the source of transmission remains unknown. One month after recovery, she started a full vaccination course with tetanus-diphtheria-pertussis (Tdap) combination vaccine. In her follow-up visits she remained free of symptoms and in good physical condition, while her chest X-ray returned to normal.

\section{Discussion}

The classical description of the clinical course of pertussis includes three stages: catarrhal, paroxysmal, and convalescent. These stages are met in the majority of the cases. Still, in older or immunized patients, some attenuation of symptoms (especially of paroxysmal stage) can be observed [8]. In adults, the clinical picture may be atypical and the predominant symptom is the prolonged cough, which, in 70 to $90 \%$ of the cases, is paroxysmal in nature [4]. This atypical clinical course often results in the underdiagnosis or misdiagnosis of the disease in adults, as postviral cough, asthma, or chronic sinusitis $[9,10]$. Delay in the diagnosis can be proved hazardous, given that almost 1 in 4 adults will present complications, such as pneumonia (10\%), pneumothorax, rib fracture, hernia, seizures, and syncope, among others [11].

The gold standard method for the establishment of the diagnosis is the isolation of the pathogen from cultured tissues or fluids, mainly nasopharyngeal swabs, aspirates, or washes. Yet, several confounding factors can influence the reliability of the method, such as treatment with antibiotics, the stage of the disease, and previous vaccination history [12]. Detection of bacterial nucleic acids through Polymerase Chain Reaction (PCR) is an alternative method, which is preferable in school-age children, adolescents, and adults, with a sensitivity varying between 10 and 30\% [13]. Serology may also be useful, particularly in cases where nasopharyngeal samples for culture and PCR are not taken early in the course of infection, due to lack of suspicion of pertussis. A 4 -fold increase in anti-PT IgG with 4-6-week intervals is probably the most reliable serologic test [6]. Alternatively, a single measurement reaching an anti-PT IgG level of $>94-110 \mathrm{EU} / \mathrm{mL}$ has been suggested as the diagnostic cutoff point for recent infection [14]. Several commercial assays for measuring IgG anti-PT are available in the European Union [7], and some of these have been tested and found to be appropriate as compared to an in-house ELISA [15]. On the other hand, there is no commercial kit approved by the United States Food and Drug Administration (FDA) for pertussis diagnosis. Notably, as happened in the presented case, leukocytosis is generally uncommon in adolescents, adults, and partially immunized children and even in the absence of it, the diagnosis of pertussis should not be excluded [16]. 
Antibiotic agents of choice for pertussis treatment are macrolides, such as erythromycin, clarithromycin, and azithromycin. Appropriate antibiotic treatment can eliminate Bordetella pertussis from the respiratory tract and, consequently, prevent transmission to susceptible contacts. Furthermore, it has been proved that antibiotics decrease the probability of secondary bacterial infections and reduce duration and severity of symptoms, when given early in the course of the disease [17].

Of major interest, our patient's natural infection in childhood had been treated with erythromycin. Unfortunately, we were not able to document the exact timing of antibiotic treatment. Still, it has been suggested in the literature that macrolides administration can weaken the subsequent immune response to infections, if given early during the course of the disease [18]. Therefore, this macrolides effect may have potentially contributed to our patient's susceptibility to pertussis reinfection.

Newborns are vulnerable to infection during the first weeks of their life, given that the quantity of maternal antibodies transferred is, in most cases, insufficient to provide protection [11]. Relevant studies suggest that adult household members, including parents and grandparents, can be identified as the source of the microorganism transmission to children in approximately $22 \%$ of the cases [19]. Therefore, early identification of diseased adults is of great value, since it can lead to the effective termination of pertussis' chain of infection. Tdap boost vaccination for subjects older than 11 years is an effective prevention strategy and, therefore, should not be omitted.

It has been estimated that nearly 10 to $20 \%$ of adults with cough lasting more than a week, suffer from pertussis [20]. Similar symptoms can be caused by other pathogens, as well, including adenoviruses, respiratory syncytial viruses (RSV), human parainfluenza viruses, influenza viruses, Mycoplasma pneumonia, and rhinoviruses [9]. Coinfections, particularly with Bordetella pertussis and RSV, are commonly seen among infants [13]. In adult patients, it is essential that the differential diagnosis of persistent cough should include primary and secondary pulmonary malignancies, and imaging with Xray or Computed Tomography (CT) of the chest must be accordingly performed in these cases [21].

\section{Conclusion}

This report aims to highlight the fact that pertussis is not only a disease of the childhood, but it should be also suspected in adults, presenting with chronic cough, even if they do have a previous history of natural infection or vaccination. Prompt recognition and appropriate therapy of adult patients can result in the effective control of the symptoms, prevention of severe complications, and spread of the infection to children; thus, they are of great clinical and public health importance.

\section{Conflicts of Interest}

The authors declare that there are no conflicts of interest regarding the publication of this paper.

\section{References}

[1] N. Benamrouche, H. T. Maamar, M. Lazri et al., "Pertussis in north-central and northwestern regions of Algeria," Journal of Infection in Developing Countries, vol. 10, no. 11, pp. 1191-1199, 2016.

[2] G. P. Marconi, L. A. Ross, and A. L. Nager, "An upsurge in pertussis: Epidemiology and Trends," Pediatric Emergency Care, vol. 28, no. 3, pp. 215-219, 2012.

[3] M. Y. Chow, G. Khandaker, and P. McIntyre, "Global childhood deaths from Pertussis: a historical review," Clinical Infectious Diseases, vol. 63, pp. S134-S141, 2016.

[4] J. Y. Hong, "Update on pertussis and pertussis immunization," Korean Journal of Pediatrics, vol. 53, no. 5, pp. 629-633, 2010.

[5] Y. H. Choi, H. Campbell, G. Amirthalingam, A. J. van Hoek, and E. Miller, "Investigating the pertussis resurgence in England and Wales, and options for future control," BMC Medicine, vol. 14, no. 121, 2016.

[6] J. M. Glanz, D. L. McClure, D. J. Magid et al., "Parental refusal of pertussis vaccination is associated with an increased risk of pertussis infection in children," Pediatrics, vol. 123, no. 6, pp. 1446-1451, 2009.

[7] N. Miyashita, Y. Kawai, T. Yamaguchi, and K. Ouchi, "Evaluation of serological tests for diagnosis of Bordetella pertussis infection in adolescents and adults," Respirology, vol. 16, no. 8, pp. 1189-1195, 2011.

[8] M. H. Ebell, C. Marchello, and M. Callahan, "Clinical diagnosis of bordetella Pertussis infection: a systematic review," The Journal of the American Board of Family Medicine, vol. 30, no. 3, pp. 308-319, 2017.

[9] E. L. Hewlett and K. M. Edwards, "Pertussis: not just for kids," New England Journal of Medicine, vol. 352, no. 12, pp. 1215-1222, 2005.

[10] E. S. Bamberger and I. Srugo, "What is new in pertussis?" European Journal of Pediatrics, vol. 167, no. 2, pp. 133-139, 2008.

[11] G. Amirthalingam, H. Campbell, F. K. Norman, M. Ramsay, E. Miller, and etal., "Sustained effectiveness of the maternal Pertussis immunization program in England 3 years following introduction," in Clinical Infectious Diseases, vol. 63, pp. 236243, 2016.

[12] A. M. Wendelboe and A. Van Rie, "Diagnosis of pertussis: a historical review and recent developments," Expert Review of Molecular Diagnostics, vol. 6, no. 6, pp. 857-864, 2006.

[13] M. Riffelmann, M. Littmann, C. Hülße, W. Hellenbrand, and C. H. Wirsing Von König, "Pertussis: Not only a disease of childhood," Deutsches Arzteblatt, vol. 105, no. 37, pp. 623-628, 2008.

[14] A. L. Baughman, K. M. Bisgard, K. M. Edwards et al., "Establishment of diagnostic cutoff points for levels of serum antibodies to pertussis toxin, filamentous hemagglutinin, and fimbriae in adolescents and adults in the United States," Clinical and Diagnostic Laboratory Immunology, vol. 11, no. 6, pp. 1045-1053, 2004.

[15] M. Riffelmann, K. Thiel, J. Schmetz, and C. H. Wirsing Von Koenig, "Performance of commercial enzyme-linked immunosorbent assays for detection of antibodies to Bordetella pertussis," Journal of Clinical Microbiology, vol. 48, no. 12, pp. 4459-4463, 2010.

[16] I. Srugo, D. Benilevi, R. Madeb et al., "Pertussis infection in fully vaccinated children in day-care centers, Israel," Emerging Infectious Diseases, vol. 6, no. 5, pp. 526-529, 2000. 
[17] D. J. Hardy, D. Vicino, and P. Fernandes, "In vitro activity of solithromycin against Bordetella pertussis, an emerging respiratory pathogen," Antimicrobial Agents and Chemotherapy, vol. 60, no. 12, pp. 7043-7045, 2016.

[18] H. C. Steel, A. J. Theron, R. Cockeran, R. Anderson, and C. Feldman, "Pathogen- and host-directed anti-inflammatory activities of macrolide antibiotics," Mediators of Inflammation, vol. 2012, Article ID 584262, 17 pages, 2012.

[19] A. M. Wendelboe, E. Njamkepo, A. Bourillon et al., "Transmission of Bordetella pertussis to young infants," Pediatric Infectious Disease Journal, vol. 26, no. 4, pp. 293-299, 2007.

[20] C. H. Wirsing Von König, S. Halperin, M. Riffelmann, and N. Guiso, "Pertussis of adults and infants," Lancet Infectious Diseases, vol. 2, no. 12, pp. 744-750, 2002.

[21] M. Weinberger and B. Lockshin, "When is cough functional, and how should it be treated?” Breathe, vol. 13, no. 1, pp. 22-30, 2017. 


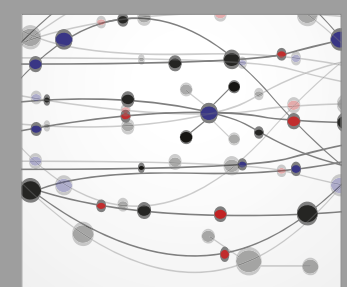

The Scientific World Journal
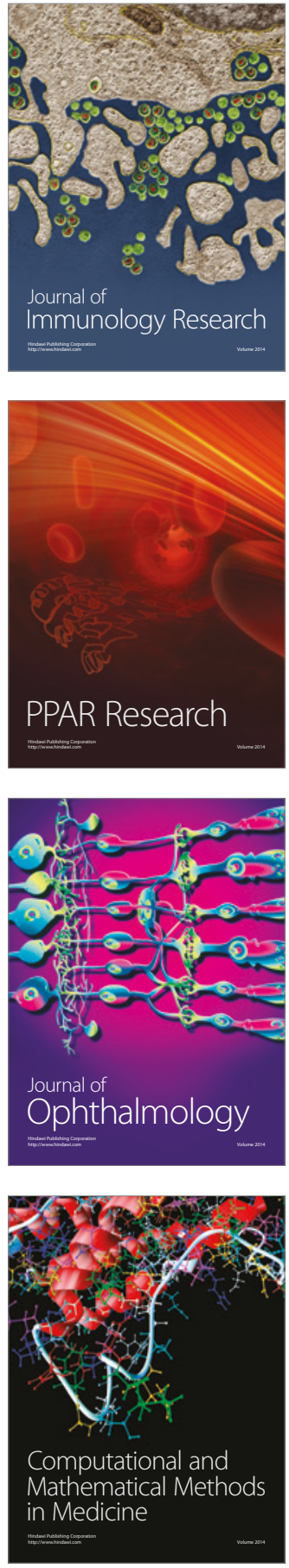

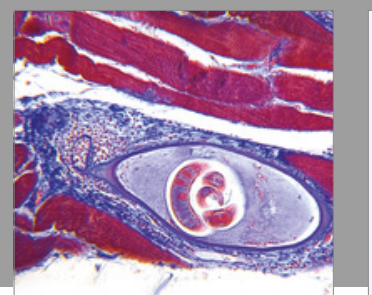

Gastroenterology Research and Practice
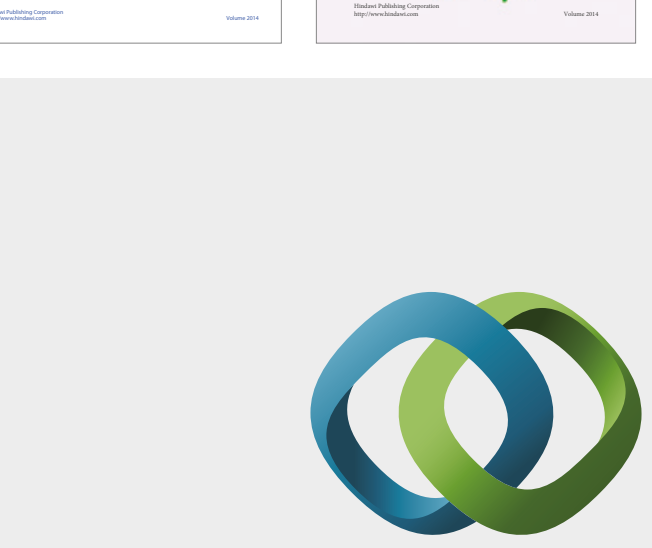

\section{Hindawi}

Submit your manuscripts at

https://www.hindawi.com
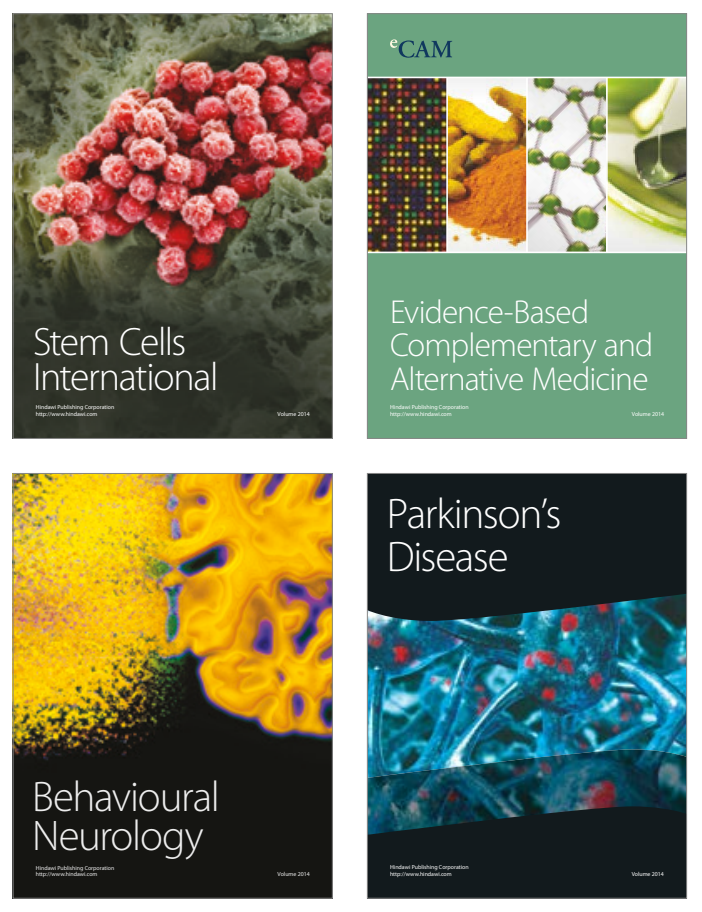
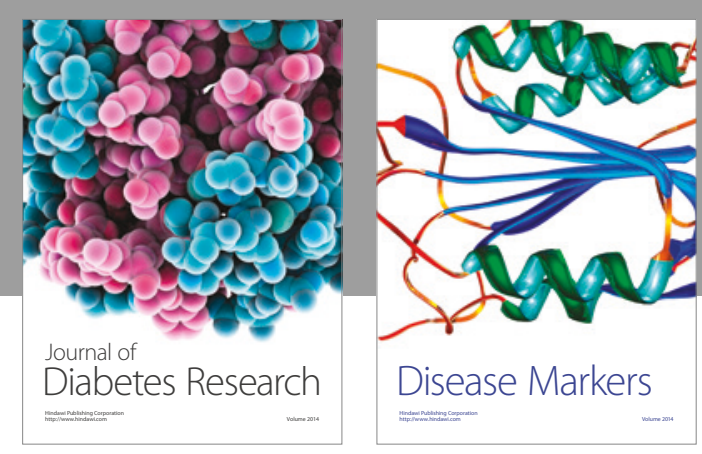

Disease Markers
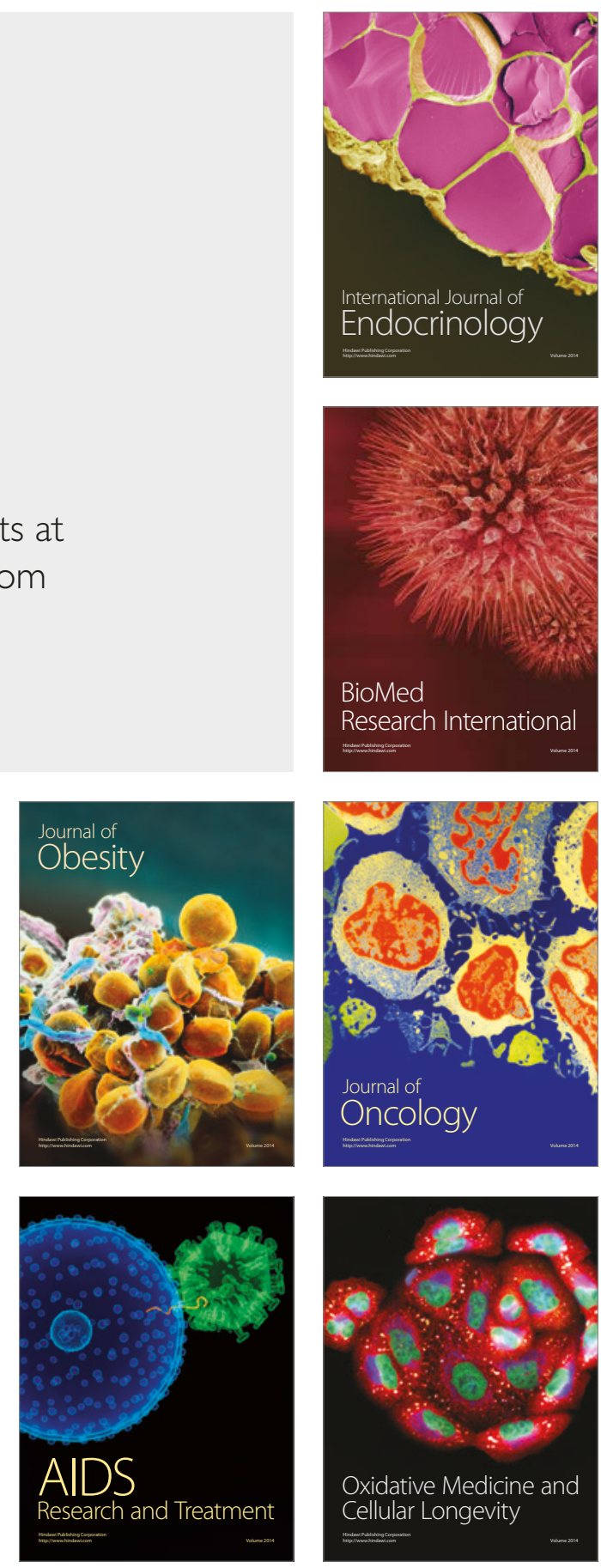